\title{
How Geometric Misalignments can affect the Accuracy of Measurements by a Novel Configuration of Self-Tracking LDV
}

\author{
Giuseppe Dinardo, Laura Fabbiano, Gaetano Vacca \\ Dept. of Mechanics, Mathematics and Management (DMMM) Politecnico di Bari, University, Bari, Italy
}

\begin{abstract}
Aim of the paper is to evaluate the reliability of a novel configuration of a Laser Doppler Vibrometer (LDV) for the measurement of vibrations affecting rotating components of machines. In the paper an analysis of errors due to the possible static misalignments of the mirrors utilized in an experimental apparatus has been carried out. This instrument was set up for the estimation of the out of plane vibrations of moving (rotating) objects, in order to give a better characterization of the self-tracking technique employed with the use of a 1D single point LDV and its measurements. The accuracies of the measures are mostly linked to the interaction between environment and instrumentation.
\end{abstract}

Section: RESEARCH PAPER

Keywords: self-tracking LDV, accuracy analysis, vibration, rotating object, geometric misalignment

Citation: G. Dinardo, L. Fabbiano, G. Vacca, How Geometric Misalignments can affect the Accuracy of Measurements by a Novel Configuration of SelfTracking LDV, Acta IMEKO, vol. 3, no. 4, article 6, December 2014, identifier: IMEKO-ACTA-03 (2014)-04-06

Editor: Paolo Carbone, University of Perugia

Received October 10 ${ }^{\text {th }}, 2013$; In final form March 24 ${ }^{\text {th }}, 2014$; Published December 2014

Copyright: @ 2014 IMEKO. This is an open-access article distributed under the terms of the Creative Commons Attribution 3.0 License, which permits unrestricted use, distribution, and reproduction in any medium, provided the original author and source are credited

Funding: (none reported)

Corresponding author: Laura Fabbiano

\section{INTRODUCTION}

An accurate evaluation of dynamic stresses due to vibrations affecting rotating machines components is crucial for both design and diagnostics interests. The accurate evaluation and determination of the dynamic stresses due to vibrations is indeed of considerable importance in the design, testing and running of turbomachinery, typically, as the measurements of these stresses become critical in the case of moving components, especially rotating ones. Reason of that is the difficulty in providing a suitable set-up capable of acquiring data from sensors placed in the same environment and transferring them to the processing unit. In order to obtain more precise measurements, some innovative nonintrusive techniques (based on the use of appropriately installed noncontact sensors) have been developed.

Nowadays, in industrial ambit, optical or electromagnetic sensors are used to characterize the vibrational state of the rotating parts of the machines, ensuring nonintrusive measurements [1].
One of the most suitable techniques is based on the use of LDV technology: a laser beam, characterized by spatial and temporal coherence, impacts on a vibrating surface; then, reflected light wave impinging the laser receiver undergoes a frequency shift (Doppler effect) allowing the evaluation of the vibrational velocity of the object or structure under examination.

The embedded difficulties in the LDV technique for the measurement of moving and vibrating organs, as turbine blades, consist in the apparent impossibility of tracking the object, from which the data required to assess the nature and the entity of vibrations have to be extracted. This is why both industrial and academic researchers focus their efforts to develop new tracking systems based on laser technology.

There is a vast literature on this subject suggesting different techniques for tracking moving objects by adding optics to the laser equipment, [2], [3], [4]. More specifically, there are several solutions involving the adoption of a Scanning Laser Doppler Vibrometer [5], [6], [7]. Nevertheless, the analysis of the errors in the measurement of vibrations by means of these configurations remains very 


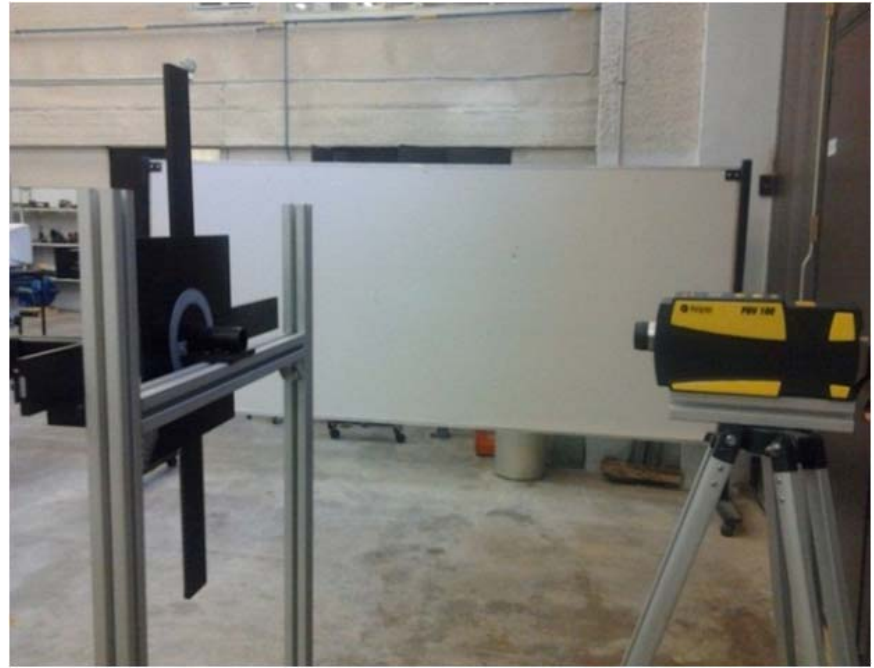

Figure 1. Mutual position between LDV and fold mirrors structure.

complex and little explored. One of those techniques has been proposed by the authors in [8], by using a 1D single point LDV apparatus. Additional equipment made by optical mirrors installed in an appropriate way is required, in order to appropriately perform the tracking of a given point lying on the rotating structure to be analysed. Other authors have analysed the geometric errors due to misalignments in the optical core system only of laser tracking configurations [9], [10], [11].

This paper deals with the study of some errors caused by the employment of additional external optics to better evaluate the relative uncertainty of the measurement. So, a qualitative analysis of uncertainties of such a configuration has been performed. These considerations are related to the static misalignments which may exist between the laser beam, the rotor axis moving the test beam and the axis of the equipment supporting the mirrors.

In this context, other possible misalignments, the so called dynamic ones due to dynamic effects induced by the movement of the object itself, are not taken into consideration.

\section{EXPERIMENTAL APPARATUS}

The experimental configuration of reference for the present analysis is the one presented in [8] and here succinctly reported in Figure 1 and in Figure 3 in the essential components.

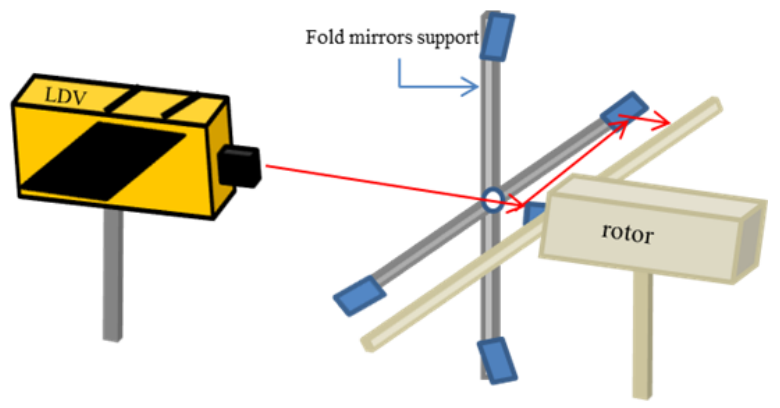

Figure 2. Experimental apparatus

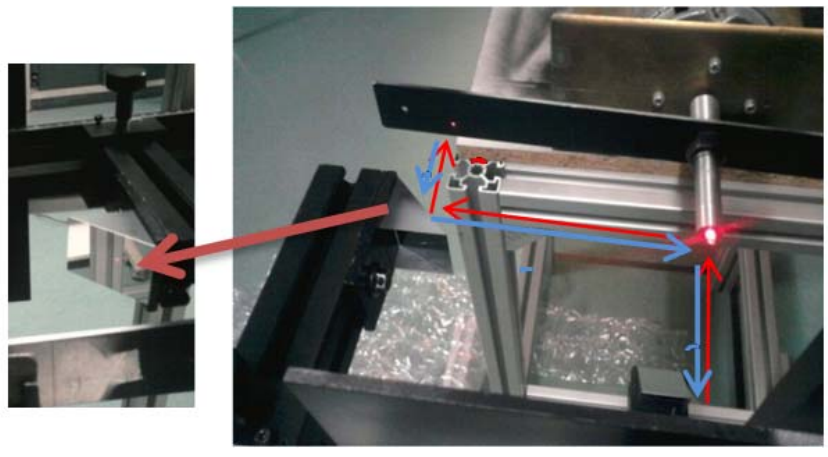

Figure 3. Laser beam paths.

Figure 1 shows the mutual position between LDV (PDV 100 Polytec) and the fold mirrors structure. The fold mirrors' support is free to rotate around its axis so that their angular position can vary.

Figure 2 shows the configuration of the experimental apparatus.

In Figure 3 the laser beam paths are simulated for easy understanding.

The fold mirrors and the vertex-mirror (rod mirror) placed on the static support and the rotor respectively are all $45^{\circ}$ mirrors (Figure 4).

\section{MISALIGNMENT ERRORS ANALYSIS}

By the LDV technique (in the self-tracking configuration) the measure of vibrations is possible at every angular velocity in principle. Nevertheless, the system can be particularly sensitive to possible static misalignments among the laser beam, the vertex mirror axis, the axis of the fold mirrors support and the rotor axis. It is immediately evident how those static misalignments cause a displacement of the measurement point on the rotating blade. As the purpose of the arranged set-up is to measure the out-of-plane vibration of a pre-set blade point while that is rotating, a perfect alignment between each pair of the setup components is required. If the alignment is not accomplished in all the four angular positions of the blade, the measurement point (where the laser spot hits) changes and thus the measurement relative to the four angular positions are not representative of the vibration of the same blade point under analysis.
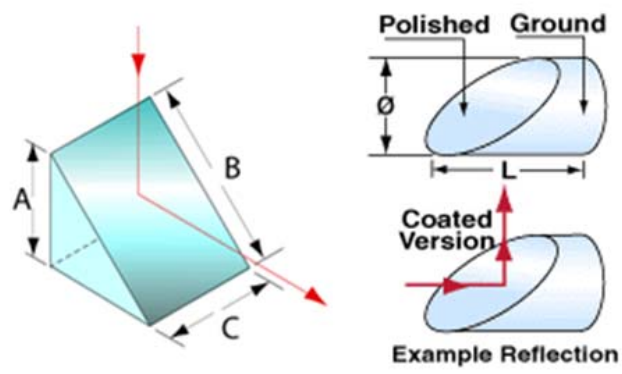

Figure 4. Technical image of the right angle mirror (fold mirror) [12] and of the $45^{\circ}$ rod mirror (vertex mirror) embedded in the rotor [13]. 
The measurement errors are mainly due to the fact that those misalignments provoke laser beam reflections not orthogonal to the incident surface. Thus, the vibration sensed is not the desired out-of-plane one and this circumstance causes additional spectral components which are superimposed to the expected measurement spectrum, causing the presence of harmonic components non representative of the original vibration velocity signal.

These complications, then, are exacerbated by dynamic effects due to the revolution, to dynamic imbalances and so on.

Although the contact absence between the blade and the sensor is an advantage (with no persistent and systematic loading effects which would influence the dynamic behaviour of the rotating structure), another source of uncertainty has to be mentioned: the vibrometer would also sense the vibration component due to the rigid body motion of the rotating structure (linked to the structural imperfections of the governor), which is superimposed to the effective and real vibration motion of the rotating object due to the fluid dynamic phenomena affecting it.

Other sources of uncertainty are certainly due to imperfections and misalignments of the optical components included into the core of the LDV head and to the output analogue signal manipulation.

\section{LDV ERRORS ANALYSIS}

The description of the frequency shift due to the Doppler effect, which provides the measure sensed by the laser beam incident onto diffusive and reflective surfaces, has well been discussed in [14] and here synthetically proposed again for clarity.

\subsection{Diffusive surface}

Several tests on diffusive surfaces have been performed in order to evaluate the amount of the errors affecting the measurements of the vibration velocity when misalignments between laser beam and surface under analysis are forced. These impositions have the main purpose of simulating misalignments which could occur in practice and due to improper installations of the components of the experimental set-up.

By theoretical considerations, these misalignments influence the frequency shift affecting the laser beam (once it is refracted by the incident surface), by a specific and fixed amount. Thus, these static misalignments (due to imprecise installation of the components of the experimental arrangement) can be treated as systematic errors.

The frequency shift of the laser beam reflected by a diffusive surface is expressed by the well-known equation: $\Delta f \cong \frac{2}{\lambda} v_{b}(t)$

where, $\lambda$ is the wavelength of the laser beam and $v_{b}(t)$ is the component of the vibration velocity vector (time dependent) along the direction of the incident laser beam.

From Equation (1), it is clear that misalignments or improper installation of the components of the experimental arrangement, influence the vibration velocity of surface under analysis.

An analysis and characterization of the errors in measuring the blade vibration velocity due to the cited effects has already been carried out in [14]. Stressing the rotating blade by a simple periodic vibration in different directions (the angles between the laser beam and the vibration velocity vector of the diffusive surface are forced), it has been shown how the induced vibrations affect its motion. The measured velocity is the component of the vibration velocity vector along the laser beam direction.

When the impressed vibration velocity onto the blade is directed along the direction of the incident laser beam, the velocity of vibration is directly given by Equation (1). In the cases where the angle between the velocity vector and the laser beam is less than $90^{\circ}$, a vibrational velocity is measured with same frequency as the first case but with reduced amplitude, in proportion to increasing angle.

If that angle is right $90^{\circ}$, no component of the velocity vector along the laser beam direction is detected, except for noise signal. So the output returns indeed a not null signal, though very small, because of the noise floor, speckle noise and so many other causes, mainly given by interfering phenomena.

By this analysis on diffusive surfaces, it has been possible to infer the following deductions:

- $\quad$ only vibrations with a velocity component along the laser beam direction give a significant output signal;

- vibrations orthogonal to the laser beam produce an output which is characterized only by noise, speckle noise and other interfering effects of difficult characterization.

\subsection{Reflecting surface}

Since the experimental set-up includes optical components (stationary fold mirror system and the rotating vertex mirror), an analysis of how reflection surfaces influence the frequency shift (due to Doppler effect) experienced by the laser beam is required.

The fold mirrors do not induce a frequency shift, since they are stationary. On the contrary, a moving mirror induces a frequency shift expressed by the formula, [14]:

$\Delta f=\frac{4}{\lambda}(\vec{v} \cdot \vec{n})(\vec{r} \cdot \vec{n})$

where $\vec{v}$ is the velocity vector of the laser spot point on the

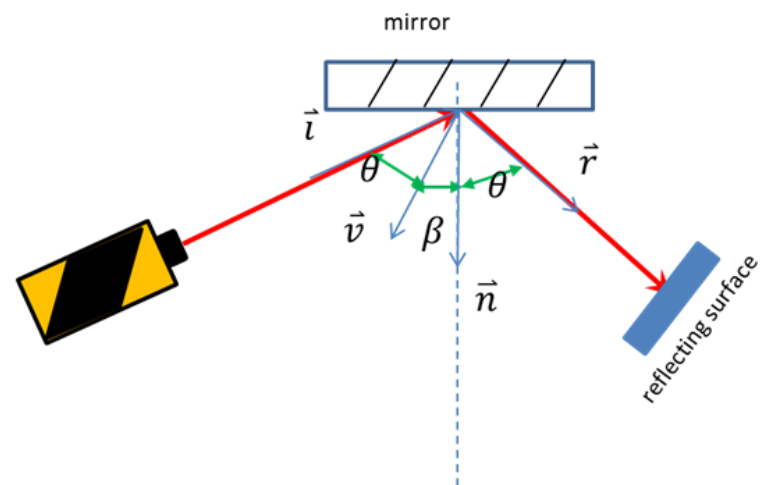

Figure 5 - General configuration for induced and reflected laser beams. 
mirror surface, $\vec{n}$ is the surface direction and $\vec{r}$ is the direction of the reflected beam, Figure 5.

In the arrangement of the self-tracking LDV, the only component giving a frequency shift is the vertex mirror, whose reflecting surface is inclined of $45^{\circ}$. It is installed on the centre of the hub supporting the blades. All the points on the reflective surface have the same angular velocity (equal to the angular velocity of the rotor). In addition each point on the reflective surface has a velocity vector which is tangential to the circular trajectory described by each of them. The point on the reflecting surface illuminated by the light spot has a velocity vector which results to be constant in time (if the angular velocity of the rotor is constant and the laser beam is stationary. This is the case here, since the fold mirror reflecting the incident laser beam is stationary). Since the reflecting surface is inclined, its normal vector will not be stationary but will rotate at the same angular velocity of the rotor and will describe a cone or a truncated cone, depending on the particular point chosen on the reflecting surface. These considerations have to be taken into account in order to quantify the frequency shift induced by the vertex mirror.

The plane containing the incident laser beam onto the vertex mirror and the reflected rotates at the same angular velocity of the rotor and is parallel to the rotation axis.

The projection of the point of the reflective surface illuminated by the laser spot and the surface of both incident and reflected laser beams vary with a sinusoidal law as the following equation states:

$v_{s}=v \cos \gamma$

where, $\vec{v}$ is the velocity vector of the point illuminated by the laser spot, $v_{s}$ its projection on the rotating surface and $\gamma$ the angle between the velocity vector, $\vec{v}$, and the surface.

Since the incident laser beam lies on a non-stationary point, it is affected by a frequency shift.

In order to avoid the frequency shift affecting the laser beam incident on the vertex mirror, a perfect alignment between laser beam, the vertex mirror and the rotor axis is required. In this way the point illuminated by the laser spot coincides with the centre of rotation of the entire system and thus, it remains stationary. No frequency shifting is then generated if the alignment is guaranteed.
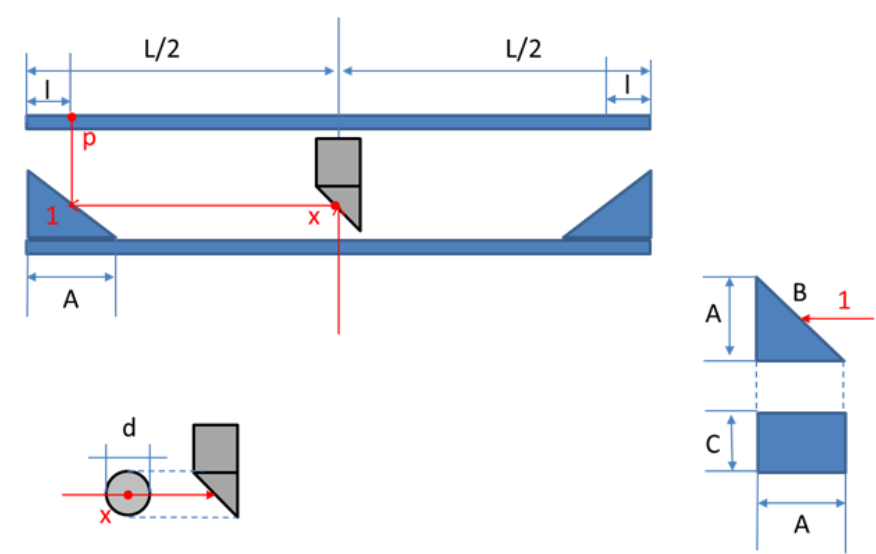

Figure 6. Perfect alignment between laser beam, vertex mirror axis and fold mirror structure

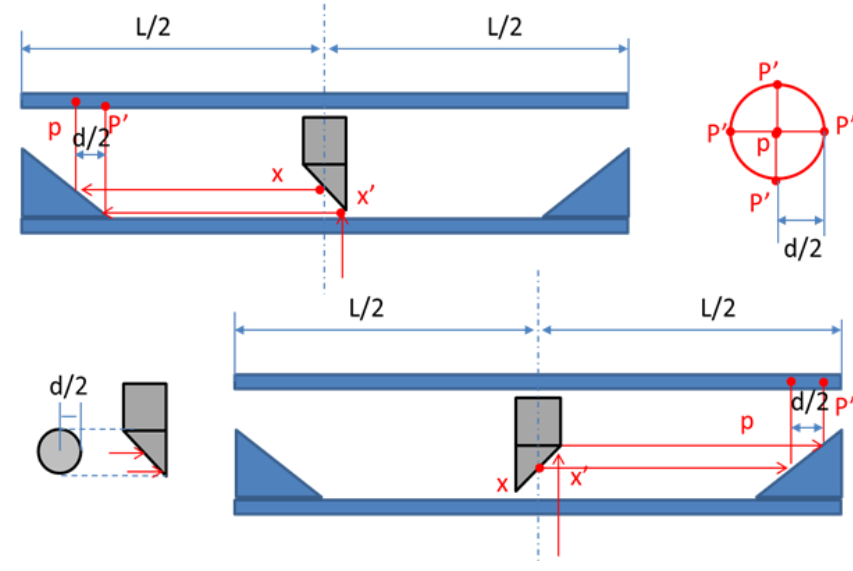

Figure 7. Parallel shift misalignment

\section{FURTHER CONSIDERATIONS ON STATIC MISALIGNMENTS}

In this paragraph, further considerations on static misalignments between laser beam and rotor and fold mirrors supporter are developed.

It is clear that these static misalignments are due to improper installation of the equipment of the described setup, leading to evaluation of vibrations of multiple points on rotating beam as far as these static misalignments cause a slip between the laser beam reflected by fold mirrors (and impinging the plane where the rotating test beam lies on).

Two types of static misalignments are going to be described in this paper: so called parallel shift misalignments and angular misalignments. The first ones occur whenever the axes of the considered components are perfectly parallel shifted and there are no angular misalignments between them.

The second ones contemplate the existence of angular misalignment between the axes.

Figure 6 represents the ideal case where a perfect alignment between laser beam, vertex mirror axis and fold mirror structure is achieved.

Figure 7 also shows the geometric distances taking place between the most considerable points in the setup.

It can be noted that a parallel shift between laser beam and vertex mirror axis results in a displacement of the
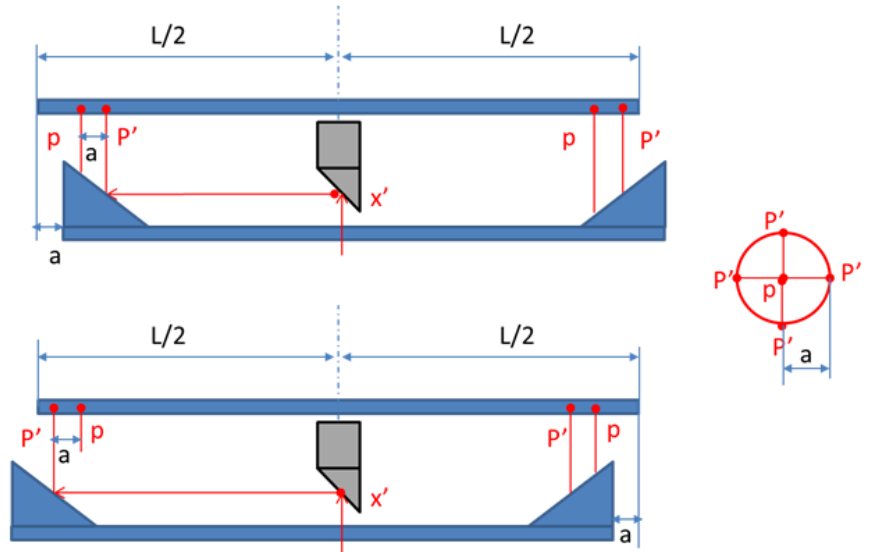

Figure 8. Parallel shift between vertex mirror axis and fold mirrors support 


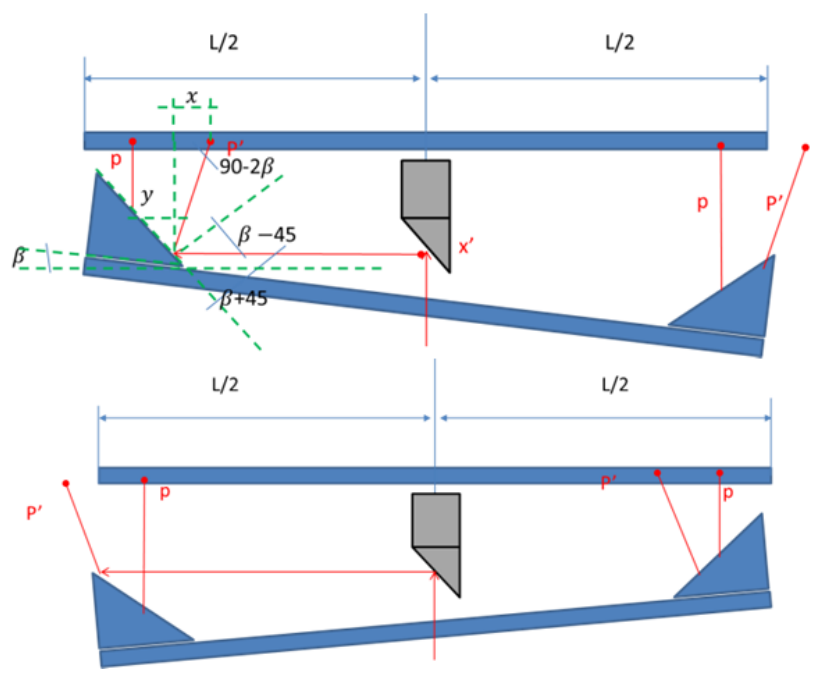

Figure 9. Angular misalignment between fold mirrors structure axis and that of the vertex mirror.

detected point on the test blade (by the laser), by an amount equal to the parallel misalignment between the laser beam and the vertex mirror axis. If we would assume that the fold mirror structure given by a cylindrical mirror with internal reflective surface inclined by 45 ', the laser beam reflected by this ideal mirror and impinging the test blade plane would be a cylinder with an axis characterized by a displacement with respect to the rotor axis equal to the amount of the parallel shift. The maximum error in tracking the effective impinging point of the laser beam onto the rotating blade is $d / 2$. A larger error would cause a complete missing of the incidence of the laser beam onto the vertex mirror. Analogous considerations could be given if we would consider a parallel shift between fold mirrors support and vertex mirror axis or a parallel shift between the former and the laser beam itself, as shown in Figure 8

The overall parallel displacement between the measuring point and the one occurring in the case of perfect alignment is given by the algebraic sum of the partial shifts occurring between the couples laser beam - vertex mirror axis and between the latter and the fold mirror support axis.

Another source of geometric uncertainty needs to be evaluated. As explained above, if the fold mirror would be the internal surface (inclined by $45^{\prime}$ ) of a cone trunk, the reflected beam successively impinging the blade plane, would be a cylinder with parallel axis and shifted to the rotor axis. Actually the fold mirror complex is formed by four mirrors with plane reflective surface. The resulting reflected beam is thus discontinuous (occurring whenever the rotating laser beam reflected by the vertex mirror impinges each fold mirror) and track segments instead of arches (because of the planarity of the fold mirror surfaces). But if the length of each fold mirror tends to zero, these segments would tend to portions of arc. This approximation would allow us to state that at each of the four angular positions (where the fold mirrors are installed), the laser point lying on the test blade would track a fixed

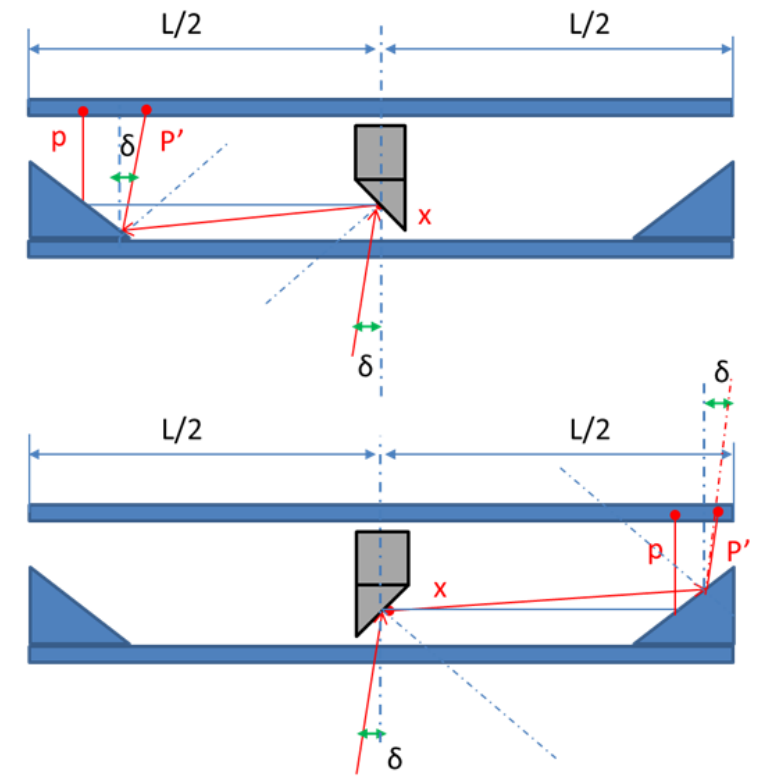

Figure 10. Angular misalignment between laser beam and rotor axis

point, but (due to parallel misalignment between laser beam - vertex mirror axis - fold mirror support axis), the point tracked at each angular position would change by an amount equal to the overall displacement between the formerly mentioned axes.

Now angular misalignments are going to be discussed.

The following picture (Figure 10) portraits these types of misalignment.

In this case, the actual measuring point is displaced by an amount proportional to $\tan (\delta)$, with $\delta$ the angular misalignment between rotor axis and laser beam and proportional to the distance between the impinging point of the laser beam on the fold mirror and the rotor plane.

Also in this case, if the fold mirror would be the internal surface (inclined by 45 ') of a cone trunk, the reflected beam successively impinging the blade plane would be a cylinder with axes inclined of $\pm \delta$ respect to the vertex mirror axis.

Since the fold mirror complex is formed by four angularly displaced mirrors with plane reflective surfaces, the reflected beams consist of a tracked segment on the blade plane, tracking more than one single point.

This would be another source of error to be neglected in the case of infinitesimal fold mirror surface length.

Figure 9 shows an angular misalignment between the fold mirror system axis and that of the rotor.

Also in this case the same consideration as above can be made.

The overall shift of the measuring point (with respect to the ideal one lying on the same point of the blade during its rotation) is given by the algebraic sum of the simpler shifts due to the stepwise angular misalignments between the several parts of the laser tracking system.

The analyzed errors due to static misalignments imply an uncertainty in the effective tracked point on the rotating test beam. One would expect that at each reflection performed by each fold mirror, the laser beam tracks the 
same point (although a relative uncertainty in which point is going to be tracked still persists). Actually due to a parallel shift (occurring in the case of parallel misalignments) and to an angular shift (occurring in the case of angular misalignments) of the portions (taking place at the reflection locations at each fold mirror) of the hypothetical cylindrical surface (if the length of reflective surface of fold mirror would tend to zero), the point tracked by the laser beam on the rotating test beam isn't the same.

\section{CONCLUSIONS}

The considerations made in the paper are effective in the case of only static misalignments.

If the proper alignment among laser beam, rotor axis, vertex mirror axis and fold mirrors support axis could be guaranteed, the laser beam generated by the LDV head would experience a frequency shift only due to out-of-plane vibrations of the plane blade under analysis due only to fluid-dynamics and rigid body motion phenomena. As it is unlikely to avoid such kind of imperfections, it is necessary to estimate at least the maximum error involved by them.

Then, given the systematic nature of such kind of errors, indeed non-perfectly evaluable, their estimation could be incorporated in the global accuracy of the equipment by means of their maximum values valuation. The rotation of the blades and other dynamic phenomena, such as dynamic unbalances of the rotor, might introduce dynamic misalignments which affect the overall frequency shift experienced by the laser beam. These so-called dynamic phenomena introduce random effects, which aren't predictable and highly difficult to quantify. Another phenomenon which theoretically could influence the measurements by the self-tracking LDV arrangement and its efficacy is the speckle noise. This phenomenon occurs when a relative motion is present between the laser beam spot and the surface under test. The phenomenon, due to the micro reflections and refractions locally distributed on the surface due to its roughness, essentially results in a noise floor which is superimposed on the vibration velocity signal and as such it can be opportunely filtered. By comparing the LDV technique with other likewise sophisticated techniques such as the interferometry, the former minimizes indeed such kind of error.

\section{REFERENCES}

[1] I. Ye Zablotskiy, A. Yu Korostelev, L. B. Sviblov, "Contactless measuring of vibrations in the rotor blades of turbines", Technical Translation FDT-HT-23, 1974, pp. 673-74.

[2] W. K Kulczyk, Q. V. Davis, "Laser Doppler instrument for measurement of vibration of moving turbine blade", Proceedings of the Institution of Electrical Engineers, vol. 120, n. 9, 1973.

[3] M. Zielinski, G. Ziller, "Noncontact vibration measurements on compressor rotor blades", Meas. Sci. Technol., 11, 2000, pp. 847-856.

[4] R. A. Lomenzo, A. J. Barker, A. L. Wicks, "Laser Vibrometry System for Rotating Bladed Disks" in proc. of the 18th IMAC, 1999, pp. 277-282.

[5] H. Dietzhausen, K. Bendel, N. Scelles, "Tracking Scanning Laser Doppler Vibrometers: Extending LaserVibrometry to Arbitrarily Moving Objects” in proc.of IMAC XXI, 2003.

[6] P. Castellini, N. Paone, "Development of the tracking laser vibrometer: Performance and uncertainty analysis", Review of Scientific Instruments, vol, 71, 2000, pp. 4639-4647

[7] M. Tirabassi, S. J. Rothberg, "Scanning LDV using wedge prisms" Optics and Laser In Engineering, 47 (3-4), 2009, pp. 454-460.

[8] G. Dinardo, L. Fabbiano, G. Vacca "Influences of Geometric Configuration on the Analysis of Uncertainties Affecting a Novel Configuration of Self-Tracking LDV" in proc of 12th IMEKO TC10, Florence, Italy, 2013, pp. 195-201.

[9] P. L. Teoh, B. Shirinzadeh, C. W. Foong, G. Alici, «The measurement uncertainties in the laser interferometry-based sensing and tracking technique», Measurement, vol. 32, n. 2, 2002, pp. 135-150.

[10] K. Umetsu, R. Furutnani, S. Osawa, T. Takatsuji, T. Kurosawa, "Geometric calibration of a coordinate measuring machine using a laser tracking system", Measurement Science and Technology, 16(12), 2005, pp. 24662472.

[11] S. Ibaraki, T. Hata, A. Matsubara, "A new formulation of laser step-diagonal measurement-two-dimensional case", Precision Engineering, vol. 33, n. 1, 2009, pp. 56-64.

[12] http://www.edmundoptics.com/optics/opticalmirrors/flat-mirrors/right-angle-mirrors/1930.

[13] http://www.edmundoptics.com/optics/prisms/specialtyprisms/45-degree-rod-lenses-mirrors/1413.

[14] R. A. Lomenzo, "Static Misalignment Effects in a SelfTracking Laser Vibrometry System for Rotating Bladed Disks”, Dissertation, Blacksburg, USA, 1998. 